\title{
MONITORAMENTO DE SAIS NA ÁGUA E NOS SOLOS IRRIGADOS DO PROJETO VEREDA GRANDE, PB ${ }^{1}$
}

\author{
Ladilson de Souza Macêdo² e Ivonete Berto Menino ${ }^{3}$
}

\begin{abstract}
RESUMO
Avaliaram-se a variabilidade temporal dos sais na água de irrigação e seus efeitos sobre os solos irrigados do Projeto Vereda Grande, em Boqueirão, PB, através de amostragem aleatória estratificada em três lotes de agricultores. Foram coletadas 66 amostras de solo e 33 de água, no período de janeiro de 1987 a dezembro de 1989, sendo que a água apresentou crescente risco de salinização ao longo do tempo, com elevados níveis de sódio e cloreto - 8,6 e 10,5 $\mathrm{mmol}_{c} \cdot \mathrm{L}^{-1}$, respectivamente. A presença de solos Bruno Não Cálcico e Litólicos na área de captação do manancial impede a melhoria da qualidade da água, predispondo os solos irrigados à alcalinização. Dentre as lavouras exploradas pelos irrigantes, apenas as capineiras são moderadamente tolerantes à concentração salina na zona radicular. A presença significativa de bicarbonato ( 1 a $\left.3,2 \mathrm{mmol}_{\mathrm{c}} \cdot \mathrm{L}^{-1}\right)$ na água restringe a prática da irrigação por aspersão devido, principalmente, às condições de alta evaporação, aridez do local e toxidez específica do sódio e cloreto em plantas sensíveis.
\end{abstract}

Palavras-chave: salinidade, sodificação, bruno não cálcico, aluviais

\section{MONITORING OF SALTS IN THE WATER AND IRRIGATED SOILS OF THE PROJECT OF VEREDA GRANDE, PB}

\begin{abstract}
The temporal variability of salts in irrigation water and its effects on the soils of the Vereda Grande Project near the town of Boqueirão, Paraíba State, Brazil, were evaluated through stratified random sampling in three farmer lots. Sixty-six soil and thirty-three water samples were collected from January 1987 to December 1989. The water presented an increasing salinity risk with time and high levels of sodium and chloride $\left(8,6\right.$ and $10,5 \mathrm{mmol}_{\mathrm{c}} \cdot \mathrm{L}^{-1}$, respectively). The presence of NonCalcic Brown and Litolic soils around the reservoir area prevents the improvement of the water quality, predisposing the irrigated soils to alkalization. Among the crops explored by the farmers, only fodder grasses are tolerant to saline concentration in the root zone. The considerable presence of bicarbonate ( 1 to $3.2 \mathrm{mmol}_{\mathrm{c}} \cdot \mathrm{L}^{-1}$ ) in the water restricts the practice of sprinkle irrigation, mainly due to conditions of high evaporation, local aridness and specific toxicity of the sodium and chloride in sensitive plants.
\end{abstract}

Key words: salinity, sodicity, non-calcic brown, alluvium

\footnotetext{
${ }^{1}$ Trabalho conduzido com recursos da EMBRAPA

${ }^{2}$ Pesquisador M.Sc. EMBRAPA/EMEPA-PB - Empresa Estadual de Pesquisa Agropecuária da Paraíba. R. Eurípedes Tavares 210,Tambiá, 58013-290, CP 275, João Pessoa, PB, Brasil. E-mail: macedo@emepa.org.br, Fone (083) 221-4504, Fax (083) 222-4971

${ }^{3}$ Eng. Agr., M.Sc./EMEPA-PB
} 


\section{INTRODUÇÃO}

O interesse de órgãos governamentais e de empresas privadas por maiores produções no setor agrícola é intensificado quando o déficit de produtos alimentares se aproxima de uma fase crítica, requerendo-se melhor utilização dos recursos naturais, na busca de exploração agrícola mais eficiente. As constantes instabilidades climáticas têm afetado a regularidade dessa produção, tornando a agricultura de sequeiro um investimento de grande risco e com visíveis reflexos para a economia, principalmente de regiões semi-áridas. Por outro lado, o caráter educativo que a agricultura irrigada impõe, induz a uma reformulação de base em todo o processo produtivo agropecuário, com extensão aos setores associativista e mercadológico.

O atual estágio de desenvolvimento das áreas públicas irrigadas na Paraíba exige a transformação da agricultura de subsistência em economia de mercado, racionalizando a sua estrutura agrária, através da introdução de novas tecnologias que permitam a utilização integral dos recursos naturais em relação à espécie vegetal mais adaptada. A adoção, por exemplo, de culturas de grande valor econômico quase sempre está associada a altas taxas de consumo d'água, da ordem de 15.000 a $30.000 \mathrm{~m}^{3} \cdot$ ha $^{-1}$ irrigado, conforme a cultura, o método de irrigação e a localização do projeto; taxas menores, no semi-árido nordestino, provocam salinização e/ou sodificação dos solos.

Segundo Rhoades (1974) dentre os principais fatores que causam a salinização, a qualidade da água de irrigação contribui efetivamente para aumentar o teor de sais na solução do solo, pois ela pode conter de $100 \mathrm{~kg}$ (água boa) a $4.000 \mathrm{~kg}$ (água imprópria) de sal em cada $1.000 \mathrm{~m}^{3}$, e é em geral, aplicada à razão de 10.000 a $15.000 \mathrm{~m}^{3} \cdot$ ha $^{-1} \mathrm{ano}^{-1}$. Consequentemente, se não houver drenagem cerca de 1,0 a 60,0 t.ha ${ }^{-1}$ de sal poderão ser adicionadas às áreas irrigadas.

O projeto de irrigação Vereda Grande, situado na microrregião do Cariri Ocidental, foi concebido para irrigar 281ha, utilizando água de classe C3-alta salinidade (Richards, 1954) desaconselhada para uso em solos com deficiência de drenagem e necessidade de práticas especiais de controle de sais, até mesmo em solos bem drenados. O projeto encontra-se, ainda, em fase de implantação. Este problema, comum a todos os mananciais do Estado da Paraíba, tem despertado o interesse da EMEPA/EMBRAPA que, dada a importância do assunto no contexto produtivo agrícola, desenvolveram ações de pesquisa no sentido de acompanhar a evolução dos sais nos solos irrigados, a fim de prevenir e/ou controlar a salinização/sodificação, freqüentemente encontrada em regiões semi-áridas, em virtude das características climáticas (alta evaporação e temperatura, baixa umidade do ar e ventos significativos) que exigem altas demandas de água ( 2 a 4 vezes a pluviometria anual) para irrigação em função do ciclo vegetativo e exigência hídrica das espécies cultivadas.

\section{MATERIAL E MÉTODOS}

O projeto de irrigação Vereda Grande (PVG) está localizado no município de Boqueirão, PB, aproximadamente a $168 \mathrm{~km}$ de João Pessoa e a $48 \mathrm{~km}$ de Campina Grande, com acesso para a cidade de Boqueirão (coordenadas geográficas: $07^{\circ} 28$ ' 40" S, $36^{\circ} 07^{\prime} 12$ " W.Gr. e $360 \mathrm{~m}$ de altitude) pela rodovia BR 104 . O município possui área de $1.127 \mathrm{~km}^{2}$, representando $24,87 \%$ da microrregião Cariri Ocidental e $2 \%$ do Estado.
A área estudada está situada na bacia do Rio Taperoá, que percorre todo o município e deságua no açude Boqueirão $\left(577,3 \times 10^{6} \mathrm{~m}^{3}\right)$.

As precipitações pluviométricas mensais ocorridas no período de 1987 a 1989 são apresentadas na Tabela 1.

Tabela 1. Precipitações pluviais no período de 1987 a 1989, no município de Boqueirão, PB

\begin{tabular}{lrrr}
\hline \multicolumn{1}{c}{ Mês } & \multicolumn{3}{c}{ Ano } \\
\cline { 2 - 4 } & 1987 & 1988 & 1989 \\
\hline Janeiro & 2,8 & 35,4 & 0,0 \\
Fevereiro & 2,0 & 30,4 & 19,0 \\
Março & 82,8 & 113,2 & 59,3 \\
Abril & 45,0 & 93,8 & 95,8 \\
Maio & 8,6 & 34,2 & 97,6 \\
Junho & 41,6 & 33,6 & 56,8 \\
Julho & 25,4 & 24,3 & 54,7 \\
Agosto & 0,0 & 26,2 & 53,3 \\
Setembro & 0,0 & 6,4 & 0,0 \\
Outubro & 0,0 & 0,0 & 0,0 \\
Novembro & 0,0 & 0,0 & 0,0 \\
Dezembro & 0,0 & 30,6 & 33,7 \\
\hline Total & 208,2 & 428,1 & 407,2 \\
\hline
\end{tabular}

De acordo com a classificação de Köppen, citada por Lima \& Heckendorff (1985) o clima é do tipo Bsh - semi-árido quente, sendo março-abril-maio o trimestre mais chuvoso, e agostosetembro-outubro o mais seco. Na classificação bioclimática de Gaussen (Galvão, 1967) corresponde a 2b (subdesértico quente de tendência tropical). A estação seca varia de 9 a 11 meses e o índice xerotérmico situa-se entre 150 e 200. A umidade relativa do ar é de $65 \%$. Os recursos naturais (água e solo) foram coletados em amostras compostas de vários locais, sempre com água corrente do rio e o solo nas profundidades de $0-20$ e $20-40 \mathrm{~cm}$, seguindo o esquema de amostragem aleatória estratificada em três pequenas propriedades rurais de irrigantes, onde foram cultivadas lavouras de milho, feijão, tomate, banana e capineiras, por iniciativa dos agricultores.

Ao término de cada ano observado foram abertas trincheiras e descritos perfis até $2,00 \mathrm{~m}$ de profundidade, visando à identificação de sais, em face da remoção diferencial de água e sais pelas plantas (Biggar et al., 1984). $\mathrm{Na}$ avaliação dos componentes da água de irrigação utilizaram-se os critérios estabelecidos por Richards (1954) segundo os quais foram analisados os parâmetros: condutividade elétrica $(\mathrm{CE}) \mathrm{em} \mathrm{dS} . \mathrm{m}^{-1}, \mathrm{pH}$, os principais cátions $\left(\mathrm{Ca}^{++}, \mathrm{Mg}^{++}, \mathrm{Na}^{+}, \mathrm{K}^{+}\right)$e ânions $\left(\mathrm{SO}_{4}^{--}, \mathrm{CO}_{3}^{--}, \mathrm{HCO}_{3}^{-}\right.$, $\mathrm{Cl}^{-}$) e a relação de adsorção de sódio ajustada (RASaj) calculada a partir da equação desenvolvida por Richards (1954) modificada para incluir os efeitos adicionais da precipitação ou dissolução do cálcio nos solos e relacionada às concentrações de carbonato e bicarbonato, que é apresentada por Scaloppi \& Brito (1986) como:

$$
\text { RASaj }=\frac{\mathrm{Na}^{+}}{\left[\left(\mathrm{Ca}^{++}+\mathrm{Mg}^{++}\right) / 2\right]^{1 / 2}}[1+(8,4-\mathrm{pHc})]
$$

onde $\mathrm{Na}^{+}, \mathrm{Ca}^{++}, \mathrm{Mg}^{++}$representam as concentrações de sódio, cálcio e magnésio, respectivamente, em mmol. $\mathrm{L}^{-1}$ de solução, e pH é o valor calculado da amostra, desenvolvido por Wilcox (1966) que avalia a tendência da água de irrigação de dissolver o cálcio do solo, aumentando ou reduzindo o cálcio solúvel, precipitando-o. $\mathrm{Na}$ interpretação da análise da água, utilizaram-se os limites 
recomendados em Califórnia University (1975) e os conceitos atualizados sobre as relações solo-água-planta apresentados por Bernstein \& François (1973) nos quais a maior parte da absorção de água ocorre nas porções superiores do sistema radicular.

No solo, avaliaram-se condutividade elétrica do extrato de saturação $(\mathrm{CEe}), \mathrm{pH}$ e porcentagem de sódio trocável (PST) e, no acompanhamento da evolução de sais em áreas irrigadas, adotou-se a metodologia de Abreu et al. (1973). As análises de água e solo foram realizadas pelo Laboratório de Irrigação e Salinidade da UFPB/Campus II.

As observações de campo foram realizadas através de trincheiras seguindo-se recomendações de Lemos \& Santos (1984).

$\mathrm{Na}$ área irrigada predominam solos Aluviais Eutróficos (Fluventes) (68\%) caracterizada por solos pouco desenvolvidos, sem horizontes genéticos, provenientes de deposições coluviais e aluvionais, normalmente estratificados, com seqüência de camadas Ap, 2C, 3C e 4C, os quais não guardam entre si qualquer relação pedogenética. São solos profundos a muito profundos, apresentando características morfológicas muito variáveis, principalmente em função da natureza dos sedimentos ocorrendo, portanto, solos que mostram os mais diferentes aspectos no que diz respeito à morfologia (textura, cor, estrutura e consistência). Os solos aluviais apresentam alta saturação de bases, com aumento gradativo em profundidade dos teores de sódio, porém se mostram de grande potencialidade agrícola na região estudada e ocupam 191ha da área total do projeto. Os Bruno Não Cálcicos compreendem solos com horizonte $\mathrm{Bt}$, textura média e/ou argilosa, Ta (alta atividade), estrutura em blocos subangulares e angulares bem desenvolvida. Esses solos apresentam pouca profundidade, variando entre 40 e $60 \mathrm{~cm}$, moderadamente drenados, com elevados teores de minerais primários de fácil intemperização, ocupando cerca de 90ha (31\%) da área total do projeto.

\section{RESULTADOS E DISCUSSÃO}

Os resultados referentes à variabilidade média das características físico-químicas da água permite enquadrá-la na classe C3 (alta salinidade) não sendo recomendada para irrigar plantas sensíveis e em solos com drenagem deficiente (Tabela 2). Verifica-se que a condutividade elétrica da água de irrigação (CEa) variou de 0,83 a 2,62 dS.m ${ }^{-1}$, sendo consideradas, segundo a Califórnia University (1975) águas que têm crescente risco de salinidade. A CEa representa uma medida da quantidade total de sais solúveis; a princípio, todas as águas e solos contêm sais e até mesmo quando as áreas utilizadas para irrigação apresentam reduzidas concentrações salinas, existe um potencial de salinização em condições de chuva e/ou drenagem; portanto, a prevenção da salinidade é tão importante quanto as ações corretivas após sua constatação.

Os valores do pH da água de irrigação estiveram dentro dos limites normais $(6,5$ a 8,4) estabelecidos por Ayers (1977); isto revela não haver necessidade de se investigar problemas afins.

Com referência aos constituintes iônicos, destacaram-se o $\mathrm{Na}^{+}$e o $\mathrm{Cl}^{-}$com concentrações médias de 8,59 a 10,53 mmol. $\mathrm{L}^{-1}$, respectivamente, cuja toxidez específica $\left(>3 \mathrm{mmol} . \mathrm{L}^{-1}\right)$ pode limitar a absorção de água pelos vegetais, através do xilema (seiva bruta) e do floema (seiva elaborada) de maneira sempre crescente, principalmente devido ao tipo de sistema de irrigação utilizado (aspersão) e à significativa presença de bicarbonato $\left(0,97\right.$ - 3,20 mmol. $\left.\mathrm{L}^{-1}\right)$ que restringe esse tipo de rega em locais de elevada evaporação.

A RASaj variou de 2,97 a 14,9 $\left(\mathrm{mmol}_{\mathrm{c}} \cdot \mathrm{L}^{-1}\right)^{0,5}$; nos dois primeiros anos de observação, os valores da RASaj permaneceram elevados indicando, segundo a classificação citada por Scaloppi \& Brito (1986) crescente e severo risco de permeabilidade. Com referência ao ano de 1989, os valores encontrados situaram-se sem nenhum problema de diminuição da permeabilidade no período de março a julho e crescente risco no mês de novembro. Esses resultados estão de acordo com Macêdo \& Santos (1992).

A diminuição da permeabilidade provoca acentuada redução na infiltração da água no solo, dificultando a reposição de lâminas d'água durante o manejo da irrigação e impede a troca de gases entre o solo e o ar atmosférico, afetando adversamente as culturas irrigadas.

Verifica-se que na área de solos Bruno Não Cálcicos a CEe teve valores mais altos apenas no primeiro ano, não se registrando nenhum valor superior a $4,0 \mathrm{dS} . \mathrm{m}^{-1}$ nos anos seguintes, nas profundidades amostradas (Tabela 3). Com referência ao $\mathrm{pH}$ do Bruno Não Cálcico, todos os valores encontram-se dentro do limite de solos normais (menor que 8,5) mesmo quando analisado conjuntamente com a CEe e PST;

Tabela 2. Variabilidade média das determinações químicas da água de irrigação do Projeto Vereda Grande, Boqueirão, PB, no período de janeiro/87 a dezembro/89

\begin{tabular}{|c|c|c|c|c|c|c|c|c|c|c|c|c|}
\hline \multirow[t]{2}{*}{ Características } & \multirow[t]{2}{*}{ Unidade } & \multicolumn{5}{|c|}{1987} & \multicolumn{3}{|c|}{1988} & \multicolumn{3}{|c|}{1989} \\
\hline & & Jan. & Abr. & Jul. & Out. & Dez. & Mar. & Jul. & Nov. & Mar. & Jul. & Nov. \\
\hline $\mathrm{CEa}^{1}$ & $\mathrm{dS} . \mathrm{m}^{-1}$ & 0,99 & 1,05 & 2,62 & 1,04 & 1,99 & 2,51 & 1,20 & 1,58 & 0,86 & 0,83 & 0,84 \\
\hline $\mathrm{PH}$ & & 8,07 & 8,15 & 8,40 & 8,16 & 8,10 & 7,70 & 7,70 & 7,80 & 7,50 & 7,40 & 7,70 \\
\hline $\mathrm{Mg}^{++}$ & $\mathrm{mmol}_{\mathrm{c}} \cdot \mathrm{L}^{-1}$ & 3,42 & 2,86 & 12,96 & 1,15 & 3,42 & 8,10 & 3,20 & 4,60 & 2,66 & 1,66 & 2,38 \\
\hline $\mathrm{Na}^{+}$ & $\mathrm{mmol}_{\mathrm{c}} \cdot \mathrm{L}^{-1}$ & 6,00 & 13,05 & 13,83 & 7,50 & 6,00 & 17,40 & 6,70 & 9,80 & 6,00 & 2,24 & 6,00 \\
\hline $\mathrm{HCO}_{3}^{--}$ & $\operatorname{mmol}_{\mathrm{c}} \cdot \mathrm{L}^{-1}$ & 1,10 & 1,03 & 1,46 & 0,97 & 1,10 & 3,20 & 2,30 & 3,10 & 1,62 & 1,57 & 1,62 \\
\hline $\mathrm{Cl}^{--}$ & $\operatorname{mmol}_{\mathrm{c}} \cdot \mathrm{L}^{-1}$ & 7,75 & 7,87 & 20,78 & 8,87 & 7,75 & 20,9 & 8,50 & 11,90 & 7,47 & 6,90 & 7,12 \\
\hline $\mathrm{SO}_{4}^{--}$ & & Pres. & Pres. & Pres. & Pres. & Pres. & Pres. & Pres. & Pres. & Aus. & Aus. & Aus. \\
\hline RASaj & $\operatorname{mmol}_{\mathrm{c}} \cdot \mathrm{L}^{-1}$ & 6,87 & 8,67 & 8,08 & 8,94 & 8,87 & 14,9 & 8,10 & 10,9 & 5,79 & 2,97 & 6,78 \\
\hline
\end{tabular}

${ }^{1}$ Águas de classe $\mathrm{C}_{3}$ (alta salinidade) não recomendadas para irrigação de plantas sensíveis e em solos com drenagem deficiente

$\mathrm{CEa}=$ Condutividade elétrica da água de irrigação 
Tabela 3. Variabilidade média dos parâmetros de salinidade do solo Bruno Não Cálcico no Projeto Vereda Grande, PB, no período 1987 a 1989

\begin{tabular}{|c|c|c|c|c|c|c|c|c|c|c|}
\hline Parâmetros & $\begin{array}{l}\text { Profundidade } \\
\quad(\mathrm{cm})\end{array}$ & \multicolumn{3}{|c|}{1987} & \multicolumn{3}{|c|}{1988} & \multicolumn{3}{|c|}{1989} \\
\hline $\mathrm{CEe}$ & $20-40$ & 3,84 & 0,54 & 4,80 & 2,30 & 1,24 & 1,54 & 1,32 & 0,97 & 2,52 \\
\hline \multirow[t]{2}{*}{$\mathrm{pH}$} & $0-20$ & 6,70 & 7,70 & 7,90 & 6,70 & 7,01 & 5,90 & 6,70 & 6,50 & 6,50 \\
\hline & $20-40$ & 7,80 & 7,88 & 8,10 & 7,20 & 7,35 & 6,00 & 6,00 & 5,50 & 6,50 \\
\hline PST & $0-20$ & 3,07 & 3,90 & 4,13 & 3,85 & 4,40 & 2,44 & 2,20 & 1,93 & 3,81 \\
\hline
\end{tabular}

$\mathrm{CEe}=$ Condutividade elétrica do extrato de saturação do solo $\left(\mathrm{dS} . \mathrm{m}^{-1}\right)$

PST $=$ Porcentagem de sódio trocável

Tabela 4. Variabilidade média dos parâmetros de salinidade de Aluvissolos do Projeto Vereda Grande, PB, no período 1987 a 1989

\begin{tabular}{|c|c|c|c|c|c|c|c|c|c|c|}
\hline \multirow[t]{2}{*}{ Parâmetros } & \multirow{2}{*}{$\begin{array}{l}\text { Profundidade } \\
(\mathrm{cm})\end{array}$} & \multicolumn{3}{|c|}{1987} & \multicolumn{3}{|c|}{1988} & \multicolumn{3}{|c|}{1989} \\
\hline & & Jan. & Jul. & Dez. & Jan. & Jul. & Dez. & Jan. & Jul. & Dez. \\
\hline \multirow[t]{2}{*}{ CEe } & $0-20$ & 2,76 & 2,02 & 2,76 & 0,89 & 0,50 & 0,85 & 0,65 & 0,37 & 0,79 \\
\hline & $20-40$ & 2,89 & 2,52 & 2,64 & 1,03 & 0,82 & 0,82 & 0,60 & 0,56 & 0,66 \\
\hline \multirow[t]{2}{*}{$\mathrm{pH}$} & $0-20$ & 7,30 & 7,21 & 7,50 & 7,20 & 7,90 & 7,30 & 7,80 & 7,50 & 7,60 \\
\hline & $20-40$ & 7,00 & 6,93 & 7,80 & 6,70 & 7,60 & 7,40 & 7,90 & 7,70 & 7,60 \\
\hline \multirow{2}{*}{ PST } & $0-20$ & 3,80 & 4,20 & 4,01 & 3,75 & 2,82 & 3,85 & 4,35 & 4,00 & 3,90 \\
\hline & $20-40$ & 3,87 & 4,54 & 3,85 & 4,15 & 2,92 & 4,15 & 3,80 & 3,95 & 4,21 \\
\hline
\end{tabular}

$\mathrm{CEe}=$ Condutividade elétrica do extrato de saturação do solo $\left(\mathrm{dS} . \mathrm{m}^{-1}\right)$

PST $=$ Porcentagem de sódio trocável

verifica-se que não há tendência à sodificação. Nos meses de julho e dezembro do ano de 1987, em que as médias do pH continuam elevadas, a PST permaneceu com valores menores que 15, induzindo-lhe ligeira salinidade. A CEe, nesse mesmo período, assumiu valores médios superiores a 4,0 dS.m ${ }^{-1}$. Nesses solos a lixiviação do excesso de sais solúveis modifica acentuadamente as propriedades físicas, tornando-as semelhantes às dos sódicos.

Através dos resultados da variação das características de avaliação da salinidade nos solos Aluviais (Ae) apresentados na Tabela 4, verifica-se que os valores registrados para $\mathrm{CEe}, \mathrm{pH}$ e PST estão dentro dos limites para solos não-salinos, de acordo com Richards (1954)

Com base nos resultados e nas análises química e física dos perfis dos solos Aluviais (Tabela 5) e do Bruno Não Cálcico (Tabela 6) pode-se considerar que, em geral, as limitações associadas aos solos aluviais estão relacionadas à ocorrência de sódio e sais solúveis em profundidades abaixo de $1 \mathrm{~m}$, porém não afetam culturas tradicionalmente exploradas na área (milho, feijão, melancia e tomate). Pelas diversas variações nas características dos solos Aluviais que ocorrem na área estudada, podem ser irrigados pelos métodos localizados em função das camadas arenosas superficiais, da baixa capacidade de retenção de água ao longo do perfil e da alta demanda evaporativa. Os

Tabela 5. Análises química e física do Aluvissolo (Ae) de textura arenosa a média e relevo plano, correspondentes à Unidade Ae1, do Projeto Vereda Grande, Boqueirão, PB

\begin{tabular}{|c|c|c|c|c|c|c|}
\hline \multirow[t]{2}{*}{ Características } & \multirow[t]{2}{*}{ Unidade } & \multicolumn{5}{|c|}{ Profundidade $(\mathrm{cm}) /$ horizonte } \\
\hline & & $0-15 / A p$ & $15-55 / 2 \mathrm{C}_{1}$ & $55-112 / 3 \mathrm{C}_{2}$ & $112-165 / 4 \mathrm{C}_{3}$ & $165-200 / 5 \mathrm{C}_{4}$ \\
\hline Cálcio trocável & $\mathrm{cmol}_{\mathrm{c}} \cdot \mathrm{kg}^{-1}$ & 3,80 & 6,00 & 7,90 & 9,50 & 3,10 \\
\hline Magnésio trocável & $\mathrm{cmol}_{\mathrm{c}} \cdot \mathrm{kg}^{-1}$ & 2,30 & 2,20 & 3,90 & 5,20 & 2,70 \\
\hline Sódio trocável & $\mathrm{cmol}_{\mathrm{C}} \cdot \mathrm{kg}^{-1}$ & 0,30 & 0,70 & 0,97 & 0,83 & 1,21 \\
\hline Potássio disponível & $\mathrm{cmol}_{\mathrm{c}} \cdot \mathrm{kg}^{-1}$ & 0,35 & 0,12 & 0,15 & 0,12 & 0,25 \\
\hline Alumínio trocável & $\mathrm{cmol}_{\mathrm{c}} \cdot \mathrm{kg}^{-1}$ & 0,02 & 0,00 & 0,00 & 0,00 & 0,00 \\
\hline Matéria orgânica & $\%$ & 0,91 & 0,79 & 0,66 & 0,62 & 0,28 \\
\hline Fósforo assimilável & $\mathrm{cmol}_{\mathrm{c}} \cdot \mathrm{kg}^{-1}$ & 4,72 & 4,41 & 2,23 & 1,84 & 1,13 \\
\hline $\mathrm{pH}\left(\mathrm{H}_{2} \mathrm{O}(1 ; 2,5)\right.$ & - & 6,90 & 7,00 & 7,00 & 7,20 & 7,80 \\
\hline CE extrato de aturação & dS. $m^{-1}$ & 1,90 & 2,50 & 1,60 & 1,70 & 2,20 \\
\hline PST & $\%$ & 4,36 & 7,76 & 7,51 & 5,30 & 16,67 \\
\hline Areia & $\%$ & 84,90 & 83,10 & 56,30 & 54,90 & 85,70 \\
\hline Silte & $\%$ & 5,20 & 7,60 & 20,80 & 20,60 & 6,90 \\
\hline Argila & $\%$ & 9,90 & 9,30 & 22,90 & 24,50 & 7,20 \\
\hline Densidade Real & g. $\mathrm{cm}^{-3}$ & 2,62 & 2,60 & 2,61 & 2,62 & 2,60 \\
\hline Capacidade de Campo & $\%$ & 7,90 & 7,80 & 17,40 & 19,10 & 6,90 \\
\hline Ponto de Murcha & $\%$ & 4,00 & 4,20 & 9,10 & 10,10 & 3,60 \\
\hline
\end{tabular}


Tabela 6. Análises química e física do Bruno Não Cálcico (Nc) de textura franco argilosa, relevo suave ondulado do Projeto Vereda Grande, Boqueirão, PB

\begin{tabular}{|c|c|c|c|}
\hline \multirow[t]{2}{*}{ Características } & \multirow[t]{2}{*}{ Unidade } & \multicolumn{2}{|c|}{ Profundidade $(\mathrm{cm}) /$ horizonte } \\
\hline & & $0-20 / \mathrm{Ap}$ & $20-60 / \mathrm{Bt}$ \\
\hline Cálcio trocável & $\mathrm{cmol}_{\mathrm{c}} \cdot \mathrm{kg}^{-1}$ & 14,80 & 11,70 \\
\hline Magnésio trocável & $\mathrm{cmol}_{\mathrm{c}} \cdot \mathrm{kg}^{-1}$ & 10,60 & 17,30 \\
\hline Sódio trocável & $\mathrm{cmol}_{\mathrm{c}} \cdot \mathrm{kg}^{-1}$ & 0,32 & 0,37 \\
\hline Potássio disponível & $\mathrm{cmol}_{\mathrm{c}} \cdot \mathrm{kg}^{-1}$ & 0,25 & 0,21 \\
\hline Alumínio trocável & $\mathrm{cmol}_{\mathrm{c}} \cdot \mathrm{kg}^{-1}$ & 0,06 & 0,10 \\
\hline Matéria orgânica & $\%$ & 13,03 & 1,40 \\
\hline Fósforo assimilável & $\mathrm{cmol}_{\mathrm{c}} \cdot \mathrm{kg}^{-1}$ & 12,30 & 10,50 \\
\hline $\mathrm{pH}\left(\mathrm{H}_{2} \mathrm{O}\right)(1: 2,5)$ & & 6,70 & 6,10 \\
\hline CE extrato de saturação & $\mathrm{dS} . \mathrm{m}^{-1}$ & 0,35 & 0,54 \\
\hline PST & $\%$ & 1,15 & 1,11 \\
\hline Areia & $\%$ & 50,20 & 51,10 \\
\hline Silte & $\%$ & 21,20 & 22,40 \\
\hline Argila & $\%$ & 28,60 & 36,50 \\
\hline Densidade real & g.cm ${ }^{-3}$ & 2,59 & 2,62 \\
\hline Capacidade de campo & $\%$ & 25,30 & 29,90 \\
\hline Ponto de murcha & $\%$ & 12,40 & 14,40 \\
\hline
\end{tabular}

métodos de irrigação por superfície (sulco, inundação) são contra-indicados devido às perdas consideráveis de água e à intensa lixiviação, características desses solos.

Com referência à área de solos Bruno Não Cálcico, pela sua pouca profundidade, consistência, susceptibilidade à erosão, presença de calhaus e matacões na superfície do solo e boa capacidade de retenção de água, irrigações leves com baixa freqüência (turnos de rega mais espaçados) em plantas de sistema radicular superficial (tomate, algodão, feijão, milho) semeadas em curvas de nível, se adequam às características desse solo.

\section{CONCLUSÕES}

1. A água utilizada para irrigação apresentou crescente risco de salinização, independentemente da intensidade das chuvas, e contém elevados níveis de sódio $\left(8,6 \mathrm{mmol}_{\mathrm{c}} \cdot \mathrm{L}^{-1}\right)$ e cloreto $(10,5$ mmol. $\left.\mathrm{L}^{-1}\right)$.

2. A presença significativa de bicarbonato (1 a $\left.3,2 \mathrm{mmol} . \mathrm{L}^{-1}\right)$ na água restringe a utilização da irrigação por aspersão, devido principalmente à condição local de aridez e à toxicidade específica do sódio e cloreto, em plantas sensíveis.

3. Os solos estudados não apresentaram tendência à alcalinidade causada pelo sódio da água de irrigação.

\section{REFERÊNCIAS BIBLIOGRÁFICAS}

ABREU, T.A.S.; MILLAR, A.A.; PEREIRA, J.R. Metodologia para o acompanhamento da evolução de problemas de sais em áreas irrigadas. In: III SEMINÁRIO BRASILEIRO DE IRRIGAÇÃOEDRENAGEM. Fortaleza: DNOCS/ABID, 1975, 12p.

AYERS, R.S. Quality of water for irrigation. Journal of Irrigation and Drainage, New York, v.103, p.135-154, 1977.

BERNSTEIN, L.; FRANÇOIS, L.E. Leaching requirements studies: sensitivity of alfafa to irrigation and drainage waters. Soil Science Society of America Proceedings, Madison, v.37, p.931-993, 1973.

BIGGAR, J.W.; ROLSTON, D.E.; NIELSEN, D.R. Transport of salts by water. Califórnia Agriculture, v.18, n.10, p.10-11, 1984.

CALIFÓRNIA UNIVERSITY. Committee of Consultants. Guidelines for interpretation of quality of water for irrigation. Davis-Califórnia, 1975. 13p.

GALVÃO, M.V. Regiões bioclimáticas do Brasil. Revista Brasileira de Geografia, Rio de Janeiro, v.29, n.1. p.3-36, 1967.

LEMOS, R.C.; SANTOS, R.D. Manual de descrição de solos no campo. Rio de Janeiro: Sociedade Brasileira de Ciência do Solo/EMBRAPA/SNLCS, 1984.45p.

LIMA, P.J. de; HECKENDORFF, W.D. Climatologia. In: Secretaria de Educação do Estado da Paraíba. Atlas geográfico do Estado da Paraíba, João Pessoa, 1985. 100p.

MACÊDO, L. de S.; SANTOS, J.B. dos. Efeito da aplicação de água salina sobre os solos irrigados na bacia Sucuru/Sumé, PB. Pesquisa Agropecuária Brasileira, Brasília, v.27, n.6, p.915-922, jun. 1992.

RHOADES, J.D. Drainage for salinity control. In: VAN SCHILFGAARDE, J. (Ed.). Drainage for Agriculture. Madison: American Society of Agronomy, 1974. 700p. (Agronomy, 17).

RICHARDS, L.A. Diagnosis and improvement of saline and alkali soils. Washington: U.S. Salinity Laboratory Staff, USDA 1954. 160p. (Agriculture Handbook, 60).

SCALOPPI, E.J.; BRITO, R.A.L. Qualidade de água para irrigação. Informe Agropecuário, Belo Horizonte, v.12, n.139, p.80-94, 1986.

WILCOX, L.V. Tables for calculating pH values of water, Riverside, CA: U.S. Salinity Laboratory, 1966. Mimeo. 